\title{
Evaluation of face recognition system in heterogenous environments (visible vs NIR)
}

\author{
Debaditya Goswami \\ d.goswami@surrey.ac.uk
}

\author{
Chi Ho Chan \\ c.chanesurrey.ac.uk
}

\author{
David Windridge \\ d.windridgeesurrey.ac.uk
}

\author{
Josef Kittler \\ j.kittler@surrey.ac.uk \\ Centre for Vision Speech and Signal Processing, \\ University of Surrey Guildford
}

\begin{abstract}
Performing facial recognition between Near Infrared (NIR) and visible-light (VIS) images has been established as a common method of countering illumination variation problems in face recognition. In this paper we present a new database to enable the evaluation of cross-spectral face recognition. A series of preprocessing algorithms, followed by Local Binary Pattern Histogram (LBPH) representation and combinations with Linear Discriminant Analysis (LDA) are used for recognition. These experiments are conducted on both NIR $\rightarrow$ VIS and the less common VIS $\rightarrow$ NIR protocols, with permutations of uni-modal training sets. 12 individual baseline algorithms are presented. In addition, the best peforming fusion approaches involving a subset of 12 algorithms are also described.
\end{abstract}

\section{Introduction}

Traditional face recognition systems have been quite successful in solving problems in restricted scenarios, where the images for use are captured in controlled conditions. However, it is of greater interest, especially in the context of security applications, to investigate situations where face images are obtained in a variety of conditions.

One manifest disadvantage of face recognition systems in the visible light (VIS) spectrum is that of illumination variation[1]. The varying energy distribution and directionality of a light source, coupled with the 3D structure of the human face can lead to differences in shading and shadows. Such conditions result in rather extensive variations even between images of the same subject. The problem becomes particularly serious when the intra-subject differences exceed inter-subject variations.

The use of Near Infrared (NIR), usually described as lying in the $800-1000 \mathrm{~nm}$ range, in face-recognition to counter illumination variation has become well-established $[23,7,22,9]$. The advantages over facial images recorded in the visible light spectrum are that indirect illumination can be largely eliminated. This is due to the fact that when capturing images in the NIR spectrum, it is very unlikely that there are multiple sources of NIR illumination present.

However, uni-modal face recognition systems (VIS only or NIR only) assume that both the gallery and probe set are captured in the same spectrum. For a truly robust automated face recognition system, it is highly desirable to successfully identify a human subject regardless of the spectrum the image is captured in.

As a result, cross-spectral face biometrics has recently gained a lot of attention. Unfortunately, the experiments conducted on most of the proposed systems are not fully representative because they are either running in closed-set experiments or involving small datasets with customised protocols. The most challenging aspect of cross-spectral matching is the lack of an open database with standard potocols for system evalation.

In this paper, we introduce a database for NIR-VIS face recognition, and also specify the standard protocols to enable systematic evaluation. A series of benchmark algorithms to establish a baseline performance for NIR-VIS face recognition systems is also presented.

The contributions of this paper are two-fold. Firstly, a database with a standard protocol for the research community to facilitate a fair evaluation of cross-spectral macthing systems is provided. In addition, evaluation results for a series of benchmark systems is presented which provide a useful insight into the merits of various face matchers and fusion methods. 


\section{Literature Review}

A major issue with matching NIR-VIS is due to the variation in the optical properties of human skin across spectra[3][16][11]. There have been various approaches to tackle the difference between spectra. Most problems approach this situation with the gallery set comprising of VIS images, and the probe set consisting of NIR images.

A common approach is to use inter-spectral image pairs to learn a subspace projection for recognition. Lin et al. [10] propose the Common Discriminant Feature Extraction (CDFE) model, which is used to project NIR and VIS images into a common space for classification. On the other hand, in [21], the gallery and probe image pairs are first projected into their respective, separate PCA/LDA subspaces. These subspace projections are then used to train a Canonical Correlation Analysis (CCA) model and recognition is performed on these CCA vectors.

Overfitting is a potential shortcoming of this approach, since any enrolled subjects not in the training set will degrade the system performance in the testing stage. In an attempt to address this problem encountered by [21], the PCA/LDA models in $[8,6]$ are learned using a training set comprising both NIR and VIS images.

In addition to subspace models, one can also apply image processing techniques on cross-spectral images. These methods attempt to extract canonical information from the face pairs, such that their intrinsic feature similarity is enhanced while simultaneously reducing the inter-spectral differences. In [8, 20], the authors peform Difference of Gaussian (DoG) or Laplacian of Gaussian (LoG) filtering on the NIR images prior to feature extraction. In general, the preprocessing and subspace projection methods are combined to create a hybrid solution to the cross-spectral face matching problem.

In a novel approach to the problem, NIR images are used to synthesize a corresponding VIS image. These synthesized VIS images are then matched against a gallery set for recognition, as seen in $[2,18]$. One of the benefits of this approach is to facilitate recognition for a human observer.

A summary of existing approaches is seen in Table 1

\begin{tabular}{cclc}
\hline Preprocessing & Feature Extraction & Matching & Reference \\
\hline DoG & MBLBP $(\mathrm{H})$ & LDA (Using NIR+VIS) & {$[8]$} \\
DoG & Raw & LDA(NIR)/(VIS) + CCA(NIR+VIS) & {$[8,21]$} \\
DoG & Raw & PCA(NIR)/(VIS) + CCA(NIR+VIS) & {$[8,21]$} \\
None & Raw & CDFE (Using NIR+VIS) & {$[8,10]$} \\
None & Raw & LDA (NIR+VIS) & {$[6]$} \\
None & Raw & PCA (NIR+VIS) & {$[6]$} \\
None & LGBP, G_WLD, P LBP & KL distance & {$[12]$} \\
LoG + Binarisation & Binary img & Regional Hamming Distance & {$[20]$} \\
None & LBPH, HoG & Random subspace (ED) OR SRC & {$[5]$} \\
\hline \hline
\end{tabular}

Table 1. A summary of the state of the art techniques detailing preprocessing, feature extraction and synthesis approaches to Cross Spectral Face Recognition in the NIR-VIS domain

When comparing the performance of these algorithms, it is difficult to fairly evaluate them, since the experiments are conducted in different datasets and protocols. Although attempts have been made to establish a baseline method for each experiment, the parameters for these baseline methods have not been standardised.

\begin{tabular}{ccccc}
\hline Total Subjects & Vis Images & Nir Images & Database & Systems \\
\hline 202 & 2095 & 3002 & HFB [6] & {$[18,21,8,5]$} \\
48 & 192 & 192 & TINDERS [19] & {$[12]$} \\
50 & 100 & 300 & Custom & {$[2]$} \\
\hline
\end{tabular}

Table 2. Summary of Heterogeneous Face Image Databases (NIRVIS)

\subsection{Motivation}

Referring to Table 2, the TINDERS dataset and the Custom dataset in [2] contain frontal face images with at most 500 samples. The HFB dataset on the other hand, contains slightly deviated face images in both modalities. In addition, a subset of the VIS images contain non-uniform illumination sources.

Table 2 clearly shows that the evaluation of various systems is carried out on specific databases, thereby limiting the scope of experimental comparison. For instance, the evaluation of [12] cannot be directly compared with the other systems. It would be beneficial if a database, for example - HFB, was used to establish benchmark performance of all heterogenous NIR-VIS algorithms. However, the systems that have already been evaluated on the HFB database do not share common experimental protocols. In addition, not all the experiments are conducted on the complete database. All these factors make system performance difficult to compare directly.

In this paper, we not only introduce a database for NIRVIS face recognition but we also define a protocol which will allow for effective evaluation of subsequent systems.

\section{Database}

The aim of this database is to provide an open platform for systematic evaluation of Cross Spectral Face matching. To this end a comprehensive set of NIR-VIS facial image pairs at a series of deviated poses defined by pitch and yaw angles were captured.

The data acquisition rig consisted of a series of vertical pillars set up around a central turntable as seen in Figure 1. Each pillar contained a series of immediately adjacent NIRVIS cameras at several different heights. The subject was positioned in the centre of the rig, and the turntable rotated by increments of approximately 10 degrees. The intention was to capture a comprehensive series of NIR-VIS facial image pairs at a series of deviated poses (including frontal) in terms of both pitch and yaw. The data was captured in the following manner: 
- For each turntable angle starting at -70 degrees and ending at +60 degrees:

- For each camera starting with $\mathrm{C} 1$ and ending with $\mathrm{C} 7$ :

- Grab image with the current infrared camera.

- Grab image with the current visual camera.

- Increment turnable angle with 10 degrees.

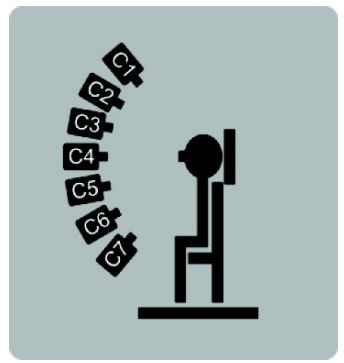

Figure 1. Database capture set up, showing the different heights of the cameras. For each $\mathrm{C} 1-\mathrm{C} 7$, a pair of adjacent NIR-VIS cameras were fitted.

Attempts were made while collecting the dataset to constitute a realistic sampling across e.g. age, gender and ethnic origin (at least $20 \%$ of non-European origin). Most importantly, the dataset was to include subjects with dark skin since visual face recognition systems have had problems with dark skin in the past, and it is unknown how the performance of infrared systems will be affected. Figure 2 shows a sample of the various subjects in the database, from a variety of ethnic backgrounds.

The complete dataset consists of 430 subjects in total, over multiple sessions. For the purposes of the experiments described in this paper, a subset of images was used with the pitch varying from $-10 / 0 /+10$ and the yaw set to a frontal

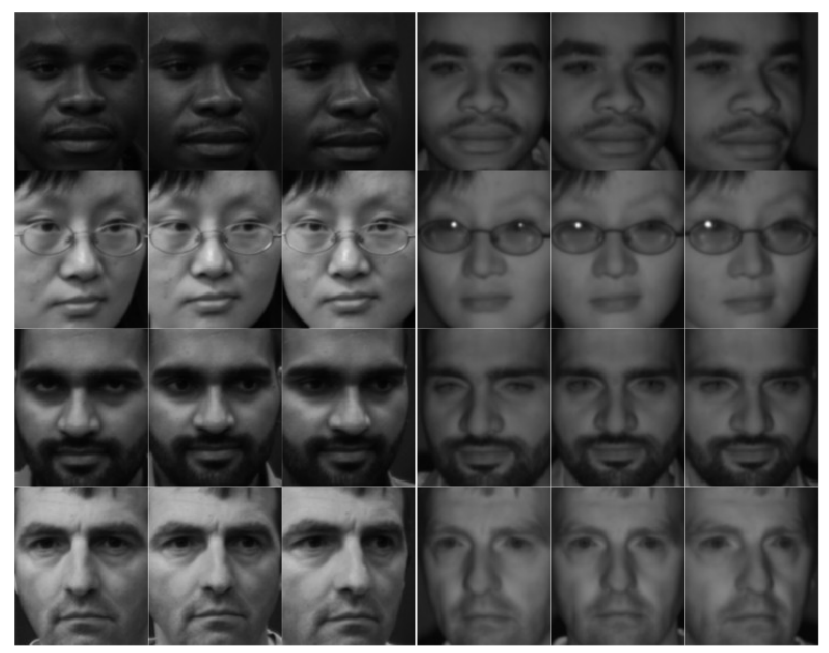

Figure 2. Images from the dataset with the pose-reflection (10/0/+10) for both VIS (left) and NIR (right)

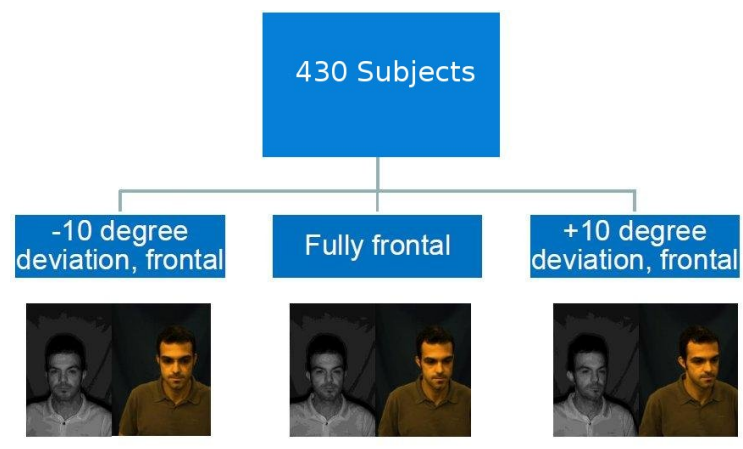

Figure 3. Dataset format

height as seen in Figure 3.

Figure 4 shows the distribution of the dataset with respect to samples:subjects. Some subjects have more than 2 samples/pose, which is down to recalibration of the rig at different capture sessions. In total there were 2103 NIR images and 2086 VIS images, with each subject containing at least 1 set of 3 poses $(-10 / 0 /+10)$.

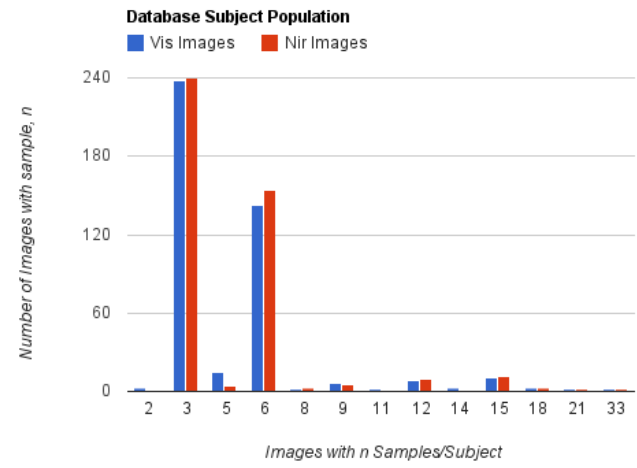

Figure 4. Database image population

Protocol: The dataset was split into training and testing subsets. To establish the training sets, the number of subjects with 6 or more images were identified and only 3 of those images were assigned for training. This was to enable some degree of overlap between training and test images. It should be noted that the NIR and VIS training sets contain a few dissimilar subjects:

- $\boldsymbol{V}_{t r n}$ the training set of 175 VIS subjects each with image poses(-10/0/+10) - 525 images .

- $\boldsymbol{N}_{t r n}$ the training set of 186 NIR images each with image poses (-10/0/+10) - 558 images.

- $V_{t s t}$ the test set of 1545 VIS images

- $\boldsymbol{N}_{t s t}$ the test set of 1563 NIR images 
Table 3 outlines the protocols used for evaluation on this database. Configurations $\mathrm{I}_{a}$ and $\mathrm{II}_{a}$, where the probe images from the NIR domain are matched against VIS gallery images, is the most commonly evaluated scenario in crossspectral matching.(e.g Immigration checkpoints, driving license checks etc.) Configuration $\mathrm{I}_{b}$ and $\mathrm{II}_{b}$ are specified for situations where it may be desirable to use a VIS probe image against a gallery of NIR images (e.g. comparing surveillance footage from a VIS source against an NIR gallery of images).

\begin{tabular}{ccccc}
\hline Configuration & Probe & Gallery & Training & Testing \\
\hline $\mathrm{I}_{a}$ & NIR & VIS & $\boldsymbol{V}_{t r n}$ & $\boldsymbol{V}_{t s t}+\boldsymbol{N}_{t s t}$ \\
$\mathrm{I}_{b}$ & VIS & NIR & $\boldsymbol{N}_{t r n}$ & $\boldsymbol{V}_{t s t}+\boldsymbol{N}_{t s t}$ \\
\hline $\mathrm{II}_{a}$ & NIR & VIS & $\boldsymbol{V}_{t r n}+\boldsymbol{N}_{t r n}$ & $\boldsymbol{V}_{t s t}+\boldsymbol{N}_{t s t}$ \\
$\mathrm{II}_{b}$ & NIR & VIS & $\boldsymbol{V}_{t r n}+\boldsymbol{N}_{t r n}$ & $\boldsymbol{V}_{t s t}+\boldsymbol{N}_{t s t}$ \\
\hline
\end{tabular}

Table 3. Protocol for the dataset

Configuration I specifies that any features are projected using the model learned on a single modality. This scenario can be used to test the robustness of the system in cases where it is not easy to acquire data to train the subspace projection models for both modalities. This scenario has not been extensively evaluated to date.

Configuration II, on the other hand specifies that images from both modalities are used to train the projection models. This enables testing of existing cross spectral algorithms which require the presence of both modalities to train the subspace projection models (like CCA).

\section{Experimental Methodology}

The face recognition system used in this paper is composed of 3 main stages- Photometric Normalisation, Feature Extraction and Classification.

\subsection{Photometric Normalisation:}

Photometric normalisation can play an important role in cross-spectral face recognition systems. The aim of this stage is to extract canonical information for each crossspectral image pair, while still retaining intrinsic features so that the inter-subject variation is not diminished. In this paper, 3 such preprocessing techniques are used.

Sequential Chain Preprocessing (SQ): consists of a series of photometric preprocessing algorithms outlined by [15]. The data is preprocessed using the following chain of algorithms:

- Gamma correction: This algorithm enhances the local dynamic range of the image in darker sections, while compressing it in bright regions and at highlights.
- Difference of Gaussian Filtering: Essentially a bandpass filter, DoG filtering removes non-essential shading from face images while still retaining the broader, more distinguishable features.

- Contrast Equalisation: Global rescaling of the image intensity values to ensure that there are no 'extreme' values

Single Scale Retinex (SSR): In [4], the image intensity value $\boldsymbol{I} \boldsymbol{m g}$ is the product between illumination $\boldsymbol{L}$ and reflectance $\boldsymbol{R}$ where the illumination component is estiminaed as a blurred original image. Therefore, the reflectance image can be estimated by substracting the original image to the estimated illumination, $\hat{\boldsymbol{L}}$,in their logarithm domains. Mathematically, SSR is described as following

$$
\begin{gathered}
\boldsymbol{R}(x, y)=\log (\boldsymbol{I} \boldsymbol{m g}(x, y))-\log (\hat{\boldsymbol{L}}(x, y)) \\
\mid \hat{\boldsymbol{L}}(x, y)=\boldsymbol{I} \boldsymbol{m g}(x, y) \otimes \boldsymbol{G}(x, y)
\end{gathered}
$$

where $G$ is an isotropic Gaussian filter.

Self Quoitent Image(SQI): The SQI [17] have been proposed for synthesizing an illumination normalised image from a face image. The SQI is defined by Img and a smoothed image $\boldsymbol{S}$ as

$$
\boldsymbol{Q}(x, y)=\frac{\boldsymbol{I} \boldsymbol{m g}(x, y)}{\boldsymbol{S}(x, y)}=\frac{\boldsymbol{I} \boldsymbol{m g}(x, y)}{\boldsymbol{I} \boldsymbol{m g}(x, y) \otimes \boldsymbol{F}(x, y)}
$$

where $\boldsymbol{F}$ is anisotropic weighted Gaussian filter.

In this work, the IN_face toolbox [14] is used to preform SSR and SQI methods. Figure 5 shows the results of different preprocessing algorithms applied.

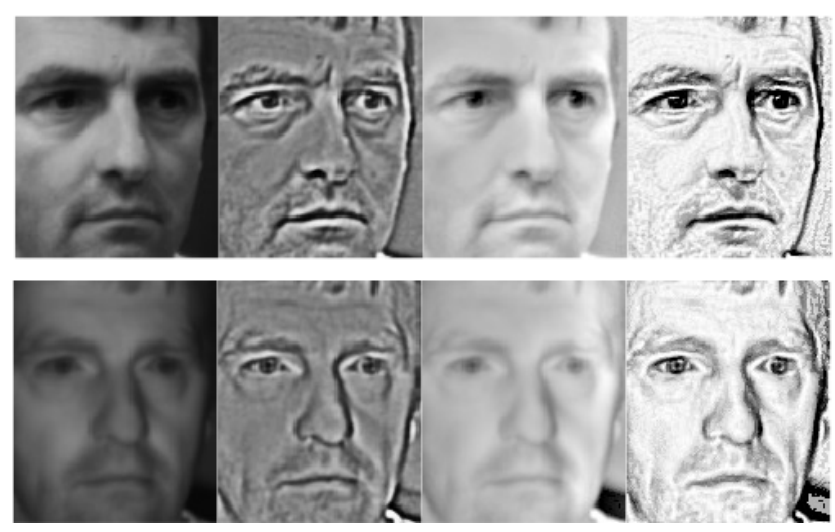

Figure 5. Image preprocessing techniques on VIS (top) and NIR (bottom). From left to right: $\mathrm{Raw} \rightarrow \mathrm{SQ} \rightarrow \mathrm{SQI} \rightarrow \mathrm{SSR}$

\subsection{Feature Extraction}

Local Binary Patterns: The LBP operator [13], shown in Equation 3, extracts information which is invariant to local 
monotonic gray-scale variations of the image. During the LBP operation, the value of current pixel, $f_{c}$, is applied as a threshold to each of the neighbours, $f_{p}(p=0, . P-1)$ to obtain a binary number. A local binary pattern is obtained by first concatenating these binary numbers and then converting the sequence into the decimal number. Using circular neighbourhoods and linearly interpolating the pixel values allows the choice of any radius, $R$, and number of pixels in the neighbourhood, $\mathrm{P}$, to form an operator.

$L B P_{P, R}(\boldsymbol{x})=\sum_{p=0}^{P-1} s\left(f_{p}-f_{c}\right) 2^{P} \quad \mid \quad s(v)= \begin{cases}1 & v \geq 0 \\ 0 & v<0\end{cases}$

In this work, uniform LBP, $L B P_{P, R}^{u 2}$, is used. Superscript $u 2$ indicates that the definition relates to uniform patterns with a $U$ value of at most 2 . If $\mathrm{U}(\mathrm{x})$ is smaller than 2 , the current pixel will be labelled by an index function, $I(z)$. Otherwise, it will assigned value $(P-1) P+2$. The index function, $I(z)$, containing $(P-1) P+2$ indices, is used to assign a particular index to each of the uniform patterns.

The resulting Pattern image is divided into nonoverlapping sub-regions, $\boldsymbol{M}_{0}, \boldsymbol{M}_{1}, . . \boldsymbol{M}_{J-1}$. The regional pattern histogram for each scale is computed as (4)

$$
\begin{gathered}
\left.\boldsymbol{h}_{j}(i)=\sum_{\boldsymbol{x} \in \boldsymbol{M}_{j}} E\left(Q_{(} \boldsymbol{x}\right)=i\right) \\
\mid i \in[0, L-1], j \in[0, J-1], \\
E(v)= \begin{cases}1 & \text { when } v \text { is true } \\
0 & \text { otherwise }\end{cases}
\end{gathered}
$$

$E(v)$ is a Boolean indicator. The set of histograms computed for each region $\boldsymbol{M}_{j}$ provides regional information while $L$ is the number of histogram bins. The classifier can directly be applied on these histograms to measure similarity of an image pair. On the other hand, a disiminative regional histogram can be computed by projecting the histogram into a linear discriminant analysis(LDA) space, as discussed in the next section.

\subsection{Dimensionality Reduction}

In order to reduce the feature dimensions and extract the disciminative information, LDA, maximising the between scatter while minimising within-scatter, is applied on the raw image space and the regional LBP histogram. A discriminative regional histogram, $\boldsymbol{d}_{l b p}^{j}$, can be obtained by projecting on the space of linear discriminant analysis(LDA). i.e., $\boldsymbol{d}_{l b p}^{j}=\left(\boldsymbol{W}_{l d a}^{j}\right)^{T} \boldsymbol{h}^{j}$, while the discriminative image feature, $\boldsymbol{d}$, is obtained by projecting the raw image feature, $\boldsymbol{f}$, into LDA space using the transformation matrix, $\boldsymbol{W}_{l d a}$. The transformation matrix, $\boldsymbol{W}_{l d a}$, is determined using training samples according to the specified protocol.

\subsection{Classification system}

In this experiment, a nearest neighbor classifer is used with two type of distance measures.

Chi-squared Histogram Distance $\left(\chi^{2}\right)$ In order to measure the similarity between two input LBP histograms resulting from a probe and an enrolled gallery image, a simple, direct measure $\operatorname{Sim}_{\chi^{2}}(\boldsymbol{G}, \boldsymbol{I})$ based on Chi-squared distance between the histograms (with bin index $i$ ) of two input videos $\boldsymbol{G}$ and $\boldsymbol{I}$ is adopted.

$$
\operatorname{Sim}_{\chi}(\boldsymbol{G}, \boldsymbol{I})=-\sum_{j} \sum_{i} \frac{\left(\boldsymbol{h}_{G}^{j}(i)-\boldsymbol{h}_{I}^{j}(i)\right)^{2}}{\boldsymbol{h}_{G}^{j}(i)+\boldsymbol{h}_{I}^{j}(i)}
$$

Normalised Correlation (NC) Normalised correlation is applied in the LDA feature space to measure the similarity between an image pair.

$$
\operatorname{Sim}_{L D A}(\boldsymbol{G}, \boldsymbol{I})=\frac{\left(\boldsymbol{d}_{G}\right)^{T} \boldsymbol{d}_{I}}{\left\|\boldsymbol{d}_{G}\right\|\left\|\boldsymbol{d}_{I}\right\|}
$$

The similarity score for the LBP approach is computed by summing the results of regional normalised correlation.

\subsection{Fusion}

In this work, a logistic regression model, $g$, is used to fuse the scores from different systems to improve the accuracy.

$$
\begin{gathered}
\operatorname{Sim}_{l o g}(\boldsymbol{G}, \boldsymbol{I})=\frac{1}{1+\exp ^{-z(\boldsymbol{v}, \beta)}}=g(\boldsymbol{v}, \beta) \\
\boldsymbol{v}=\left[\operatorname{Sim}_{0}(\boldsymbol{G}, \boldsymbol{I}), \operatorname{Sim}_{1}(\boldsymbol{G}, \boldsymbol{I}), \cdots, \operatorname{Sim}_{N-1}(\boldsymbol{G}, \boldsymbol{I})\right]^{T}, \\
z(\boldsymbol{w}, \beta)=\beta_{0}+\beta_{1} w_{0}+\cdots+\beta_{N-1} w_{N-1}
\end{gathered}
$$

where $\mathrm{N}$ is the total number of individual face system involved in fusion. To construct the model, two types of samples, called matching and non-matching sets are extracted from the training samples based on Configuration II for training. The matching set, $V_{C}=\boldsymbol{v}_{i i}{ }^{N C}$ contains the similarity scores samples of the same subject in VIS and NIR spectra and the non-matching, $V_{I}=\boldsymbol{v}_{i i}{ }^{N I}$, contains the scores samples for different subjects in VIS and NIR spectra. NC is the total number of image pairs of the same subject in both spectra and NI is the total number of image pairs for the different subjects in both spectra. To evaluate the performance of logistic regression model, the statisical toolbox of MATLAB is used.

\subsection{Baseline Experiments}

In this paper, the face images were geometrically normalised to a $142 \times 120$ resolution using manually annotated eye locations. These normalised face images were then put 
through the benchmark system. For the uniform LBPHbased systems, the LBP images were divided into $7 \times 7$ nonoverlapping regions to compute the local region histograms. Table 4 shows the list of Configuration $\mathrm{I}_{a, b}$. As can be seen, 12 experiments conducted according to the system are evaluated.

\begin{tabular}{r|c|c|c}
\hline Preprocessing & Feature & Classification & Protocol \\
\hline SQ & Image space & LDA+NC & $\mathrm{I}_{a}, \mathrm{I}_{b}$ \\
SSR & Image space & LDA+NC & $\mathrm{I}_{a}, \mathrm{I}_{b}$ \\
SQI & Image space & LDA+NC & $\mathrm{I}_{a}, \mathrm{I}_{b}$ \\
Raw & Image space & LDA+NC & $\mathrm{I}_{a}, \mathrm{I}_{b}$ \\
\hline $\mathrm{SQ}$ & Uniform LBPH & Chi-Squared & $\mathrm{I}_{a}, \mathrm{I}_{b}$ \\
$\mathrm{SSR}$ & Uniform LBPH & Chi-Squared & $\mathrm{I}_{a}, \mathrm{I}_{b}$ \\
$\mathrm{SQI}$ & Uniform LBPH & Chi-Squared & $\mathrm{I}_{a}, \mathrm{I}_{b}$ \\
Raw & Uniform LBPH & Chi-Squared & $\mathrm{I}_{a}, \mathrm{I}_{b}$ \\
\hline $\mathrm{SQ}$ & Uniform LBPH & LDA+NC & $\mathrm{I}_{a}, \mathrm{I}_{b}$ \\
$\mathrm{SQI}$ & Uniform LBPH & $\mathrm{LDA}+\mathrm{NC}$ & $\mathrm{I}_{a}, \mathrm{I}_{b}$ \\
$\mathrm{SSR}$ & Uniform LBPH & $\mathrm{LDA}+\mathrm{NC}$ & $\mathrm{I}_{a}, \mathrm{I}_{b}$ \\
Raw & Uniform LBPH & LDA+NC & $\mathrm{I}_{a}, \mathrm{I}_{b}$ \\
\hline
\end{tabular}

Table 4. Summary of Algorithms used in Configuration I

Configuration II is used to perform fusion based experiments, investigating any potential improvements in the system. With the 12 proposed systems, it is possible to obtain $4095 \times 2$ different fusion combinations for configurations $\mathrm{II}_{a}$ and $\mathrm{II}_{b}$.

\section{Results and Discussion}

\subsection{Configuration I}

Figure 6 shows the Rank 1 recognition rates for experiments conducted using configuration I. A dramatic loss in performance is seen for the raw (no preprocessing) image LDA system. This is not surprising, since the LDA projection models have been trained on one of the spectra only. This confirms the presence of an overfitting problem when the spectral composition of the images are varying to such a degree, and the necessity of an appropriate preprocessing.

Comparing the systems which incorporate preprocessing, SQ is the best of the photometric normalisations used in this paper. This further confirms the findings in Table 1 , regarding the benefits of using DoG or LoG filters as a preprocessing method. This can be attributed to the canonical manner in which SQ extracts the intrinsic features of the inter-modal image pairs.

When comparing the performance of Image space and LBPH features, the latter is seen to perform better in all cases. The reason is that the local structures for both spectra are not dissimilar. LBPH features are essentially rasterised representations of the local structure, while the image space does not capture this information.

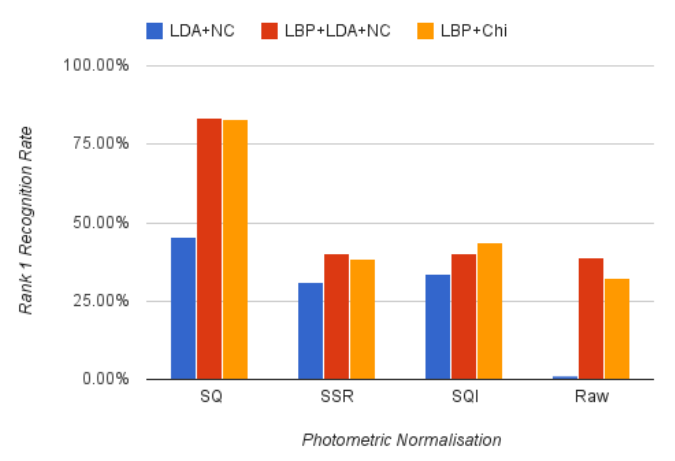

(a) Configuration $\mathrm{I}_{a}$ : NIR probe/VIS gallery

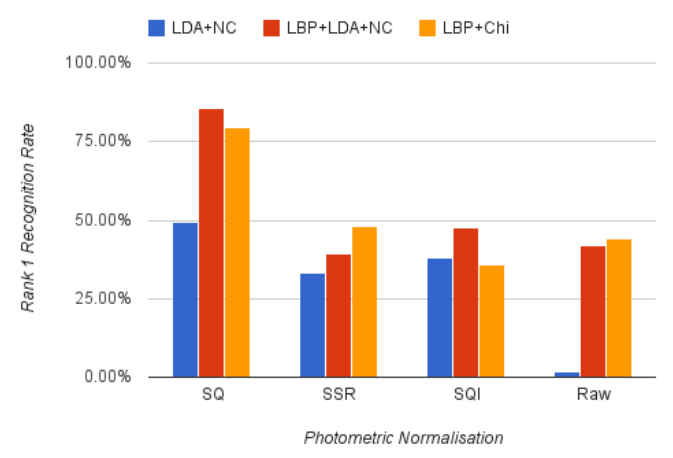

(b) Configuration $\mathrm{I}_{b}$ : VIS probe/NIR gallery

Figure 6. Rank 1 recognition rates for Configuration I

The combination of SQ+LBPH-based systems gives the best performance for both configurations $\mathrm{I}_{a, b}$. The benefits of the SQ preprocessing are highlighted again, and it can be concluded that the combination of both LBPH and SQ independently enhance the matching process.

Surprisingly, projecting the LBPH features into LDA subspace does not show a significant benefit when compared with LBPH-only system. Also, the LBPH-based systems without SQ preprocessing fail to exhibit any marked improvement in performance.

From Figure 7, it is seen that the performance of $I_{a}$ is better than $\mathrm{I}_{b}$ for all supervised learning methods (LDA). This highlights the importance of testing in both scenarios in order to obtain a true measure of system robustness.

\subsection{Configuration II}

Rank 1 recognition rates evaluating baseline experiments on configuration $\mathrm{II}_{a, b}$ are seen in Figure 8, and is similar to protocols used in previous approaches to cross-spectral matching. Also included for comparison, is the best performing combination of fused algorithms (details of which are presented later on this section) 


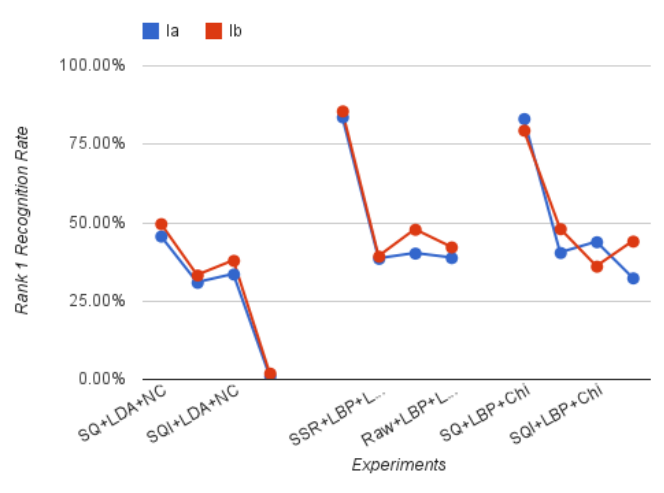

Figure 7. Analysis of Supervised and Unsupervised methods for Configuration $\mathrm{I}_{a}$ and $\mathrm{I}_{b}$

From Figure 8, it is immediately apparent that the use of CCA significantly diminishes recognition performance, when compared with the LDA classification. To investigate this, experiments on $\mathrm{II}_{a, b}$, using the training data for verifi-

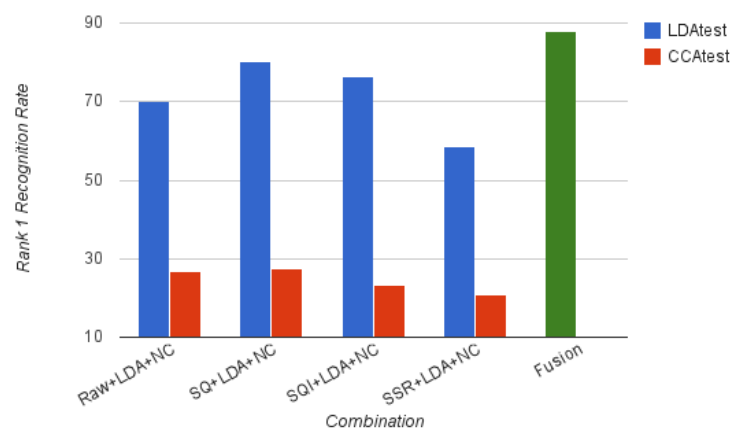

(a) Configuration $\mathrm{II}_{a}$ : NIR probe/VIS gallery

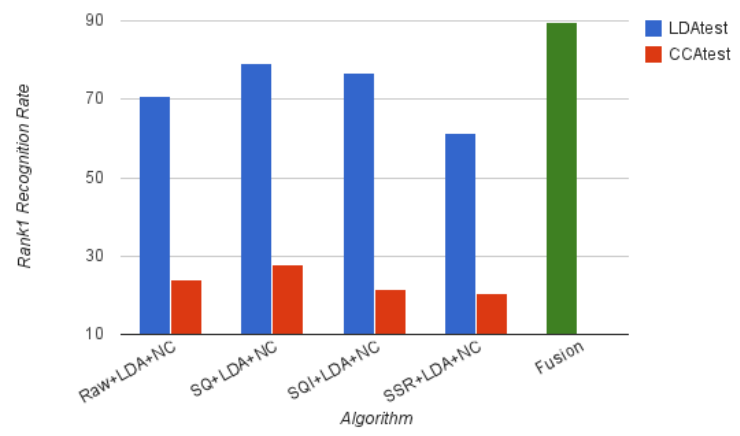

(b) Configuration $\mathrm{II}_{b}$ : VIS probe/NIR gallery

Figure 8. Rank 1 recognition rates for Configuration $\mathrm{II}_{a, b}$. NC score-matching was used for all the algorithms described cation were conducted (see Table 5).

If the system performance on training and test data is compared, it is clear that overfitting has a notable effect on the CCA-based systems. Although the LDA systems also display a drop in performance (about $10 \%$ ), the use of CCA on untrained subjects has a much more detrimental effect on the system. The negative effect of untrained subjects on CCA is briefly alluded to in [8], and can be quantitatively confirmed here.

\begin{tabular}{cccc}
\hline Configuration & Preprocessing & LDA & LDA+CCA \\
\hline \multirow{4}{*}{$\mathrm{II}_{a}$} & Raw & 76.34 & 70.76 \\
& SQ & 91.94 & 76.04 \\
& SQI & 91.04 & 74.85 \\
& SSR & 87.10 & 75.83 \\
\hline \multirow{6}{*}{$\mathrm{II}_{b}$} & Raw & 85.90 & 69.98 \\
& SQ & 97.71 & 76.02 \\
& SQI & 97.71 & 75.44 \\
& SSR & 93.90 & 75.63 \\
\hline
\end{tabular}

Table 5. Rank 1 Recognition rates conducted on Configuration II using training data

Fusion experiments were also conducted using Configuration II, where combinations of algorithms were used for evaluation, ranging from a single algorithm to 12 combined systems. Figure 9 shows the best performance in each category. It can be seen that a fusion system is the most promising in this cross spectral environment. However, when fusing more than 6 systems, the performance is seen to plateau with a downward trend as the complexity of the combinations increases.

There are 10 of 4095 different fusion combinations using $\mathrm{II}_{a}$ which reach a system-wide best performance of $87.9 \%$. These 10 fused systems are defined combinations of 5-8 individual algorithms. Using $\mathrm{II}_{b}$, there are 4 of 4095 combinations performing at $89.57 \%$. These 4 systems are defined by the combination of 6-7 algorithms.

Each of the highest performing combinations include all the SQ systems, underlining its importance in the process. In addition, it is seen that once again $\mathrm{II}_{b}$ outperforms $\mathrm{II}_{a}$. This further highlights the importance of evaluating the systems in both $a$ and $b$ configurations.

\section{Conclusion}

In this paper, we introduced a database for NIR-VIS face recognition, describing standard protocols, $\mathrm{I}_{a, b}$ and $\mathrm{II}_{a, b}$, which will enable the evaluation of both NIR $\rightarrow$ VIS and VIS $\rightarrow$ NIR face matching. The database consists of 430 subjects, each with pose deviations of $(-10 / 0 /+10)$ degrees in both VIS and NIR images, and 4189 images in total. Every attempt was made while collecting the data to incorporate subjects from a variety of ethnicities, genders and age. 


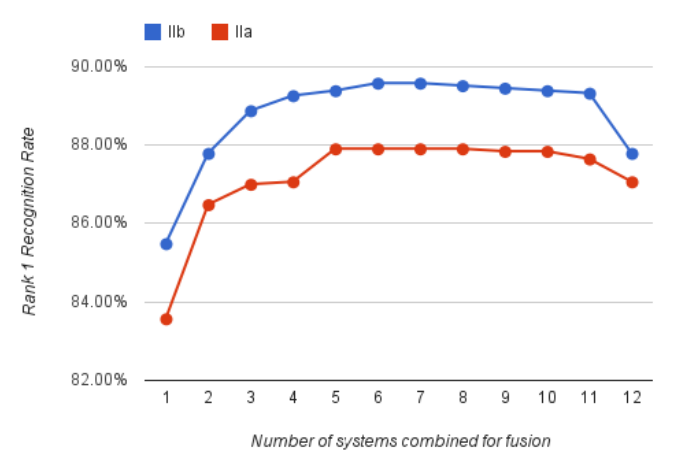

Figure 9. Rank 1 Recognition Rates for Configuration $\mathrm{II}_{a}$ and $\mathrm{II}_{b}$

A series of benchmark experiments to establish a baseline performance are also presented. It was shown that SQ preprocessing is notably beneficial in cross-spectral matching. In both configurations, there is a variability in system performance, with VIS $\rightarrow$ NIR frequently outperforming NIR $\rightarrow$ VIS. Finally, the effects of overfitting on cross-spectral face matching were shown, with demonstrating the vulnerability of CCA to such effects.

Dataset Acquisition: Please email c.chan@surrey.ac.uk, with the subject heading 'Cross Spectral Dataset'

\section{References}

[1] Y. Adini, Y. Moses, and S. Ullman. Face recognition: The problem of compensationg for changes in illumination direction. In IEEE Transactions on Pattern analysis and Machine Intelligence, 1997. 1

[2] J. Chen, D. Yi, J. Yang, G. Zhao, S. Z. Li, and M. Pietikäinen. Learning mappings for face synthesis from near infrared to visual light images. In $C V P R$, pages 156-163, 2009. 2

[3] W.-F. Cheong, S. A. PRAHL, and A. J. WELCH. A review of the optical properties of biological tissues. In IEEE Journal of Quantum Electronics, volume 26, no.2, December 1990. 2

[4] D. Jobson, Z. Rahman, and G. Woodell. Properties and performance of a center/surround retinex. Image Processing, IEEE Transactions on, 6(3):451 -462, mar 1997. 4

[5] B. Klare and A. K. Jain. Heterogeneous face recognition: Matching nir to visible light images. In International Conference on Pattern Recognition, 2009. 2

[6] S. Li, Z. Lei, and M. Ao. The hfb face database for heterogeneous face biometrics research. In Computer Vision and Pattern Recognition Workshops, 2009. CVPR Workshops 2009. IEEE Computer Society Conference on, pages $1-8$, june 2009. 2

[7] S. Z. Li, R. Chu, S. Lao, and L. Zhang. Illumination invariant face recognition using near-infrared images. In IEEE Transactions on Pattern Analysis and Machine Intelligence, volume 29, pages $627-639$, April 2007. 1
[8] S. Liao, D. Yi, Z. Lei, R. Qin, and S. Z. Li. Heterogeneous face recognition from local structures of normalized appearance. In $I C B$, pages 209-218, 2009. 2, 7

[9] S. Liao, Z.Zhu, Z.Lei, L.Zhang, and S.Z.Li. Learning multiscale block local binary patterns for face recognition. In International Conference on Biometrics, pages 828-837, 2007. 1

[10] D. Lin and X. Tang. Inter-modality face recognition. In ECCV (4), pages 13-26, 2006. 2

[11] I. V. Meglinski and S. J. Matcher. Quantitative assessment of skin layers absorption and skin reflectance spectra simulation in the visible and near-infrared spectral regions. Physiological Measurement, 23(4):741-753, 2002. 2

[12] F. Nicolo and N. A. Schmid. A method for robust multispectral face recognition. In ICIAR (2), pages 180-190, 2011. 2

[13] M. Pietikäinen, T. Ojala, J. Nisula, and J. Heikkinen. Experiments with two industrial problems using texture classification based on feature distributions. Intelligent Robots and Computer Vision XIII: 3D Vision, Product Inspection, and Active Vision, 2354(1):197-204, 1994. 4

[14] V. . Struc, T. Description, L. Thanks, I. Dr, and P. Kovesi. Inface: A toolbox for illumination invariant face recognition, 2009. 4

[15] X. Tan and B. Triggs. Enhanced local texture feature sets for face recognition under difficult lighting conditions. In Analysis and Modelling of Faces and Gestures, volume 4778/2007, pages $168-182,2007.4$

[16] T. L. Troy and S. N. Thennadil. Optical properties of human skin in the nir wavelength range of 1000-2200 nm. 2

[17] H. Wang, S. Li, Y. Wang, and J. Zhang. Self quotient image for face recognition. In Image Processing, 2004. ICIP '04. 2004 International Conference on, volume 2, pages 1397 1400 Vol.2, oct. 2004. 4

[18] R. Wang, J. Yang, D. Yi, and S. Z. Li. An analysis-bysynthesis method for heterogeneous face biometrics. In Proceedings of the Third International Conference on Advances in Biometrics, ICB '09, pages 319-326, Berlin, Heidelberg, 2009. Springer-Verlag. 2

[19] WVHTCF. Tactical imager for night/day extended-ranged surveillance. 2

[20] D. Yi, S. Liao, Z. Lei, J. Sang, and S. Z. Li. Partial face matching between near infrared and visual images in mbgc portal challenge. In M. Tistarelli and M. S. Nixon, editors, ICB, volume 5558 of Lecture Notes in Computer Science, pages 733-742. Springer, 2009. 2

[21] D. Yi, R. Liu, R. Chu, Z. Lei, and S. Z. Lei. Face matching between near infrared and visible light images. In International Conference, ICB 2007, proceedings, volume 4642/2007, pages 523 - 530, August 2007. 2

[22] S. Zhao and R. Grigat. An automatic face recognition system in the near infrared spectrum. In Proc. Intl Conf. Machine Learning and Data Mining in Pattern Recognition, pages 437-444, July 2005. 1

[23] X. Zou, J. Kittler, and K. Messer. Ambient illumination variation removal by active near-ir imaging. In D. Zhang and A. Jain, editors, Proceedings of the International Conference on Biometrics, pages 19-25. Springer, January 2006. 1 